\title{
CONVEXITY BIAS IN EURODOLLAR FUTURES PRICES: A DIMENSION-FREE HJM CRITERION
}

\author{
VLADIMIR POZDNYAKOV AND J. MICHAEL STEELE
}

\begin{abstract}
In the theory of interest rate futures, the difference between the futures rate and forward rate is called the "convexity bias," and there are are several widely offered reasons why the convexity bias should be positive. Nevertheless, it is not infrequent that the empirical the bias is observed to be negative. Moreover, in its most general form, the benchmark HJM term structure model is agnostic on the question of the sign of the bias; it allows for models where the convexity bias can be positive or negative. In partial support of the practitioner's arguments, we develop a simple scalar condition within the HJM framework that suffices to guarantee that the convexity bias is positive. Moreover, when we check this condition on the LIBOR futures data, we find strong empirical support for the new condition. The empirical validity of the sufficient condition and the periodic observation of negative bias, therefore leads one to a paradoxical situation where either (1) there are arbitrage possibilities or (2) a large subclass of HJM models provide interest rate dynamics that fail to capture a fundamental feature of LIBOR futures.
\end{abstract}

\section{INTRODUCTION}

To price contracts such as swaps which have values that are driven by the term structure of interest rates, one typically needs to know the forward LIBOR rates, but, except in isolated circumstances, these rates are not directly observable via market prices. In contrast, the Eurodollar futures give us directly observed futures LIBOR rates from actively traded contracts. Both forward and futures LIBOR rates are each in essence a kind of proxy for the spot LIBOR rate for some future date, and they differ primarily because of the daily "mark to market" conventions

\footnotetext{
Key words and phrases. Heath-Jarrow-Morton model, Eurodollar futures, convexity bias, futures rate, forward rate.

V. Pozdnyakov: Department of Statistics, University of Connecticut, 215 Glenbrook Rd, Storrs, CT 062694120; Email: vladimir.pozdnyakov@uconn.edu.

J.M. Steele: Wharton School, Department of Statistics, Huntsman Hall 447, University of Pennsylvania, 3730 Walnut Street, Philadelphia PA 19104-3603; Email: steele@wharton.upenn.edu.
} 
that govern futures contracts. Roughly put, as new information arrives the futures LIBOR rates are correspondingly adjusted while structurally the forward LIBOR rates are fixed for the entire duration of the consummated forward contract. As result, these two rates tend to move in tandem, though typically they are unequal.

The difference between the futures and forward LIBOR rates is called the convexity bias, and there is a well-known theoretical expression for this bias, say as given by Karatzas and Shreve (1998, p. 47). The formula shows that the size (and sign) of the bias is determined by the covariance between the LIBOR spot rate and a certain discount factor. Unfortunately, one cannot estimate this covariance well enough even to be confident of the sign of the bias that is implied by the formula. The problem is that vector pair consisting of the spot rate and the discount factor are highly correlated over time so that even several years of data provides very little information about the coordinate to coordinate correlation of the stationary pair. Naturally, for longer periods, one must worry about the loss of stationarity. The bottom line is that the theoretical formula for the convexity bias is not of much help when one tries to understand the empirical behavior of the convexity bias.

Fortunately, there is an alternative approach to the sign of the convexity bias that completely avoids this nearly infeasible covariance estimation. We will shortly describe a simple inner product condition that provides a sufficient condition for positive convexity bias in the context of the Heath-Jarrow-Morton term structure model.

\section{Necessary Facts about the HJM Term Structure Model}

To put the inner product condition in context, we first recall the conventional n-dimensional HJM model (Heath et al. (1992) and Musiela and Rutkowski (1997, p. 304)). Also, for the moment, we work directly under the equivalent martingale measure $P$. In particular, we assume that the dynamics of the instantaneous forward rate is given by the familiar formula:

$$
d f(t, T)=-\boldsymbol{\sigma}(t, T)^{\top} \boldsymbol{a}(t, T) d t+\boldsymbol{\sigma}(t, T)^{\top} d \boldsymbol{B}_{t},
$$

where $\left\{\boldsymbol{B}_{t}, 0 \leq t \leq \tau\right\}$ is a $n$-dimensional Brownian motion under the probability measure $P$, the adapted $n$-vector $\boldsymbol{\sigma}(\cdot, T)=\left(\sigma_{1}(\cdot, T), \sigma_{2}(\cdot, T), \ldots, \sigma_{n}(\cdot, T)\right)^{\top}$ has component processes $-\infty<\sigma_{i}(\cdot, T)<\infty$ that may be of any sign, and the process 
$\boldsymbol{a}(t, T)$ is defined by the integral

$$
\boldsymbol{a}(t, T)=-\int_{t}^{T} \boldsymbol{\sigma}(t, u) d u,
$$

This relation between the $n$-vector $\boldsymbol{\sigma}(t, u)$ and drift $-\boldsymbol{\sigma}(t, T)^{\top} \boldsymbol{a}(t, T)$ of the instantaneous forward rate is at the heart of the HJM model. Specifically, it is this connection that guarantees the absence of arbitrage between zero-coupon bonds of different maturities.

Incidentally, one should note here that while $\sigma_{i}^{2}(t, T)$ has the interpretation as an instantaneous variance, we do not restrict the sign of $\sigma_{i}(t, T)$ since to do so when $n>1$ would substantially weaken the richness of the HJM model. Thus, some care must be exercised when calling the $\sigma_{i}(t, T)$ a "volatility."

Under the HJM model the price at time $t$ of a bond that pays one dollar at the maturity date $T$ is given by

$$
P(t, T)=\exp \left(-\int_{t}^{T} f(t, u) d u\right) \quad \text { for all } 0 \leq t \leq T \leq \tau,
$$

and from this definition sees that $P(t, T)$ satisfies the SDE

$$
d P(t, T)=P(t, T)\left[r(t) d t+\boldsymbol{a}(t, T)^{\top} d \boldsymbol{B}_{t}\right]
$$

where $r(t)$ is defined by setting $r(t)=f(t, t)$. Naturally, $r(t)$ has the interpretation as the spot rate at time $t$.

The HJM model can be developed on the basis of just two fundamental assumptions: (1) the filtration of the $n$-dimensional Brownian motion is rich enough to accommodate the LIBOR term structure, and (2) the yield curve is smooth with respect to maturity $T$. If one agrees with these assumptions and absence of arbitrage one is led almost inexorably to the HJM construction.

\section{LiBOR Rates in the HJM Context}

By the $\lambda$-LIBOR rate we mean the LIBOR interest rate that is offered at time $t$ for a Eurodollar deposit for a maturity of $\lambda 360$ days. This rate is denoted by $L_{\lambda}(t)$, and it is also called the spot $\lambda$-LIBOR rate when it is useful to emphasize its distinction from the corresponding forward or futures rates. The $\lambda$-LIBOR rate is an add-on rate, so it is easily written as a function of the corresponding zero 
coupon bond:

$$
L_{\lambda}(t)=\frac{1}{\lambda}\left(\frac{1}{P(t, t+\lambda)}-1\right) \quad 0<t<\tau .
$$

The forward rate $L_{\lambda}(t, T)$ is then the interest rate that available is at time $t$ for a riskless loan that begins at date $T$ and which is paid back at time $T+\lambda$. This is also an add-on rate so it has an analogous representation as a function of zero-coupon bond prices:

$$
L_{\lambda}(t, T)=\frac{1}{\lambda}\left(\frac{P(t, T)}{P(t, T+\lambda)}-1\right) \quad 0<t<T<\tau .
$$

Finally, we will always assume that the $\lambda$-LIBOR futures rate $F_{\lambda}(t, T)$ is given by its well-known formula martingale representation formula:

(7) $\quad F_{\lambda}(t, T)=E\left[\frac{1}{\lambda}\left(\frac{1}{P(T, T+\lambda)}-1\right) \mid \mathcal{F}_{t}\right]=E\left[L_{\lambda}(T) \mid \mathcal{F}_{t}\right] \quad 0<t<T<\tau$.

Karatzas and Shreve (1998, p. 45)) provide conditions that suffice for the validity of this representation, and Pozdnyakov and Steele (2004) provide alternative conditions that seem to be more easily justified in the context of interest rate futures. Here we simply take the truth of the identity (7) to be one of our underlying assumptions, so there is need to separate out the more primitive conditions under which it is known to hold.

\section{The Futures Rate-Forward Rate Bias}

By the Futures Rate-Forward Rate Bias we mean the quantity

$$
\operatorname{Bias}_{\lambda}(t, T) \equiv F_{\lambda}(t, T)-L_{\lambda}(t, T)
$$

This bias (8) has been considered on several occasions in the financial literature (e.g. Burghardt and Hoskins (1995), Grinblatt and Jegadeesh (1996), Gupta and Subrahmanyam (2000), Henrard (2005), Piterbarg and Renedo (2006)), and these authors all note that practitioners commonly take it as an a priori truth that the bias is positive. That is, in practice it is typically assumed that for all $0<t<T$ and $\lambda$ one has the bound

$$
\operatorname{Bias}_{\lambda}(t, T)=F_{\lambda}(t, T)-L_{\lambda}(t, T) \geq 0 \quad \text { with probability one. }
$$

Nevertheless, as Burghardt and Hoskins (1995), Grinblatt and Jegadeesh (1996) and Pozdnyakov and Steele (2002) have observed in a variety of time frames, it 
is an empirical fact that this bias can be negative. Moreover, when the time to maturity $T-t$ is relatively small, say less that a year, the bias is frequently found to be negative. This situation creates both theoretical and practical issues. In particular, it now seems useful to try to understand the conditions that suffice to guarantee when an HJM model will have a consistently positive bias.

On such condition was given in Pozdnyakov and Steele (2002) where it is proved that when the forward rate diffusion equation

$$
d f(t, T)=-\boldsymbol{\sigma}(t, T)^{\top} \boldsymbol{a}(t, T) d t+\boldsymbol{\sigma}(u, T)^{\top} d \boldsymbol{B}_{t}
$$

has all components of the vector $\boldsymbol{\sigma}(\cdot, T)=\left(\sigma_{1}(\cdot, T), \sigma_{2}(\cdot, T), \ldots, \sigma_{n}(\cdot, T)\right)^{\top}$ with the same sign, then one does indeed have positive bias (9) for all $0<t<T$ and $\lambda$.

What concerns us here is that in the proof of this constant sign condition criterion for the positive bias, it was also proved as an intermediate result (pp. 185-187) that one has positive bias provided that for $0<s<U<T$ one has the bound

$$
|\boldsymbol{a}(s, U)|^{2} \leq \boldsymbol{a}(s, U)^{\top} \boldsymbol{a}(s, T) \quad \text { a.s. }
$$

In retrospect, it appears that this inner product criterion is actually more convenient than the constant sign criterion for determining when an HJM model has a positive futures-rate, forward-rate bias. The reason for this is that because of the diffusion equation for the zero-coupon bond price

$$
d P(t, T)=P(t, T)\left[r(t) d t+\boldsymbol{a}(t, T)^{\top} d \boldsymbol{B}_{t}\right]
$$

one can show that inner products of vectors of the form $\boldsymbol{a}(\cdot, \cdot)^{\top}$ can be computed from the (one-dimensional!) volatility of the bond price. This observation permits one to construct practical empirical estimates of the inner product condition (11) that do not require a full parametric specification of the driving HJM model. Moreover, one can test the condition (11) without ever having to confront thorny issue of specifying the dimensionality $n$ of the HJM model.

In passing, we should that diffusion equation for the bond price provides an intuitive interpretation of inner product criterion (11). Loosely speaking, the inner product criterion (11) asserts that the convexity bias will be positive provided that the price volatility of bonds with longer maturities is larger than the price volatility of bonds with shorter maturities. This is often, but not always, the case. 


\section{An Inner Product Approximation}

Shortly we will find that market data provides strong empirical evidence in support of inner product inequality (11). What makes such a market test feasible is that for each $\lambda_{1}$ and $\lambda_{2}$ one can approximate $\boldsymbol{a}\left(\lambda_{2}\right)^{\top} \boldsymbol{a}\left(\lambda_{1}\right)$ with help from the quadratic variation of the spot rate process $t \mapsto L_{\lambda}(t)$.

To see how this is done, first recall that the process $P(\cdot, \cdot+\lambda)$ is a diffusion that satisfies the stochastic differential equation

$$
d P(t, t+\lambda)=P(t, t+\lambda)\left[(r(t)-f(t, t+\lambda)) d t+\boldsymbol{a}(t, t+\lambda)^{\top} d \boldsymbol{B}_{t}\right],
$$

so by Itô formula we have

(12) $d L_{\lambda}(t)=\frac{1}{\lambda P(t, t+\lambda)}\left[\left(f(t, t+\lambda)-r(t)+|\boldsymbol{a}(t, t+\lambda)|^{2}\right) d t-\boldsymbol{a}(t, t+\lambda)^{\top} d \boldsymbol{B}_{t}\right]$.

Next we consider the quadratic variation $\left\langle L_{\lambda}(\cdot), L_{\lambda}(\cdot)\right\rangle_{t}$ of the spot $\lambda$-LIBOR rate, and we recall (Karatzas and Shreve (1991, pp. 32 and 138) or Steele (2001, p. 128)) that one can think about the quadratic variation in two different ways. First, it can be presented as a limit (in probability) of a discrete quadratic variation (or realized quadratic volatility) over a partition $0=t_{0}<t_{1}<t_{2}<\ldots<t_{K}=t$ :

$$
\left\langle L_{\lambda}(\cdot), L_{\lambda}(\cdot)\right\rangle_{t}=\lim _{\max _{i}\left(t_{i+1}-t_{i}\right) \rightarrow 0} \sum_{i=0}^{K-1}\left[L_{\lambda}\left(t_{i+1}\right)-L_{\lambda}\left(t_{i}\right)\right]^{2} .
$$

Second, since the spot $\lambda$-LIBOR rate $L_{\lambda}(\cdot)$ is an Itô integral, the quadratic variation $\left\langle L_{\lambda}(\cdot), L_{\lambda}(\cdot)\right\rangle_{t}$ also can be viewed as a Riemann integral:

$$
\begin{aligned}
&\left\langle L_{\lambda}(\cdot), L_{\lambda}(\cdot)\right\rangle_{t}=\int_{0}^{t}\left\{\frac{|\boldsymbol{a}(s, s+\lambda)|}{\lambda P(s, s+\lambda)}\right\}^{2} d s \\
&=\max _{i}\left(\lim _{i+1}-t_{i}\right) \rightarrow 0 \\
& \sum_{i=0}^{K-1}\left\{\frac{\left|\boldsymbol{a}\left(t_{i}, t_{i}+\lambda\right)\right|}{\lambda P\left(t_{i}, t_{i}+\lambda\right)}\right\}^{2}\left(t_{i+1}-t_{i}\right) \quad \text { a.s. }
\end{aligned}
$$

To use these representations $\boldsymbol{a}(\cdot, \cdot+\lambda)$ must be well behaved, and, to proceed heuristically for the moment, we will consider a strong (but simple) non-parametric assumption. Specifically, we assume that for all sufficiently small positive $t$ the process $\boldsymbol{a}(s, s+\lambda), 0<s<t$ is deterministic and depends only on $\lambda$. That is, we consider the case when $\boldsymbol{a}(t, t+\lambda) \equiv \boldsymbol{a}(\lambda)$ for all $s \in[0, t]$ for some small $t$. One might worry if this is too much to ask, but, with a little more work, one can check that it suffices to know that one can approximate the process $s \mapsto \boldsymbol{a}(s, s+\lambda)$ as closely as one likes by a process that is predictable and piecewise constant. 
Now, if we observe the LIBOR rate $L_{\lambda}(\cdot)$ during $K$ business days denoted by the times $0=t_{0}<t_{1}<t_{2}<\ldots<t_{K}=t$, then from the two representations for the quadratic variation we obtain

$$
\left\langle L_{\lambda}(\cdot), L_{\lambda}(\cdot)\right\rangle_{t} \approx \sum_{i=0}^{K-1}\left[L_{\lambda}\left(t_{i+1}\right)-L_{\lambda}\left(t_{i}\right)\right]^{2} \approx \frac{|\boldsymbol{a}(\lambda)|^{2}}{\lambda^{2}} \sum_{i=0}^{K-1} \frac{t_{i+1}-t_{i}}{P\left(t_{i}, t_{i}+\lambda\right)^{2}} .
$$

Therefore, taking into account that $P(t, t+\lambda)=\left(1+\lambda L_{\lambda}(t)\right)^{-1}$ we get the very instructive formula

$$
|\boldsymbol{a}(\lambda)|^{2} \approx \lambda^{2} \frac{\sum_{i=0}^{K-1}\left[L_{\lambda}\left(t_{i+1}\right)-L_{\lambda}\left(t_{i}\right)\right]^{2}}{\sum_{i=0}^{K-1}\left(t_{i+1}-t_{i}\right)\left(1+\lambda L_{\lambda}\left(t_{i}\right)\right)^{2}} .
$$

The benefit of this representation is that all the quantities on the righthand side are directly observable from market prices.

To obtain more generally a formula for $\boldsymbol{a}\left(\lambda_{2}\right)^{\top} \boldsymbol{a}\left(\lambda_{1}\right)$ we call on the quadratic cross-variation for $\lambda$-LIBOR rates of two maturities $\lambda_{1}$ and $\lambda_{2}$. In direct analogy with our first derivation, we find

$$
\begin{aligned}
\left\langle L_{\lambda_{2}}(\cdot), L_{\lambda_{1}}(\cdot)\right\rangle_{t} & \approx \sum_{i=0}^{K-1}\left[L_{\lambda_{2}}\left(t_{i+1}\right)-L_{\lambda_{2}}\left(t_{i}\right)\right]\left[L_{\lambda_{1}}\left(t_{i+1}\right)-L_{\lambda_{1}}\left(t_{i}\right)\right] \\
& \approx \frac{\boldsymbol{a}\left(\lambda_{2}\right)^{\top} \boldsymbol{a}\left(\lambda_{1}\right)}{\lambda_{2} \lambda_{1}} \sum_{i=0}^{K-1} \frac{t_{i+1}-t_{i}}{P\left(t_{i}, t_{i}+\lambda_{2}\right) P\left(t_{i}, t_{i}+\lambda_{1}\right)},
\end{aligned}
$$

and, as a consequence, we find the approximation

$$
\boldsymbol{a}\left(\lambda_{2}\right)^{\top} \boldsymbol{a}\left(\lambda_{1}\right) \approx \lambda_{2} \lambda_{1} \frac{\sum_{i=0}^{K-1}\left[L_{\lambda_{2}}\left(t_{i+1}\right)-L_{\lambda_{2}}\left(t_{i}\right)\right]\left[L_{\lambda_{1}}\left(t_{i+1}\right)-L_{\lambda_{1}}\left(t_{i}\right)\right]}{\sum_{i=0}^{K-1}\left(t_{i+1}-t_{i}\right)\left(1+\lambda_{2} L_{\lambda_{2}}\left(t_{i}\right)\right)\left(1+\lambda_{1} L_{\lambda_{1}}\left(t_{i}\right)\right)} .
$$

Here one should note that these formulas were developed with respect to the martingale measure, but, given our assumption on $a(s+\lambda, \lambda)$, they are also valid under the original measure since quadratic variation is the same in each case.

\section{Examination of the Assumptions}

When we model the term structure with help of the HJM construction we have just one (admittedly large) "free parameter" - the volatility of the instantaneous forward rate, $\{\boldsymbol{\sigma}(t, u), 0 \leq t \leq u \leq \tau\}$. Naturally, this vector-valued process must satisfy some mild regularity conditions and it must be adapted to the filtration $\mathcal{F}_{t}$, but one is still left with a vast amount of freedom.

Since $\boldsymbol{\sigma}(t, u)$ and the bond volatility $\boldsymbol{a}(t, T), 0 \leq t \leq T \leq \tau$ are related by the no-arbitrage condition (2), one may alternatively begin the HJM modeling process 
by specifying the process $\boldsymbol{a}(t, T)$ instead of the specifying the volatility $\boldsymbol{\sigma}(t, u)$ of the instantaneous forward rate. Here, of course, we have to keep in mind that because of the integral representation (2), we must choose a bond volatility $\boldsymbol{a}(t, T)$ that is smooth with respect to the second variable. Further, since our principal focus is on LIBOR, we can choose to base our model design on a choice of the $\lambda$-LIBOR rate volatility $\boldsymbol{a}_{\lambda}(t)=\boldsymbol{a}(t, t+\lambda)$ instead of the bond volatility $\boldsymbol{a}(t, T)$. To be sure, the two points of view are ultimately equivalent.

Here it seems most appropriate to focus on the LIBOR rate volatility $\boldsymbol{a}_{\lambda}(t)$. Our main assumption about $\boldsymbol{a}_{\lambda}(t)$ is that it can be well approximated by predictable piecewise constant process with respect to $t$. Specifically, we consider the LIBOR models for which there is some partition $0=T_{0} \leq T_{1} \leq \cdots \leq T_{N}=\tau-\lambda$ of $[0, \tau]$ such that

$$
\boldsymbol{a}_{\lambda}(t)=\sum_{i=0}^{N-1} \boldsymbol{a}_{\lambda}^{T_{i}} 1_{T_{i} \leq t \leq T_{i+1}} \quad \text { where } \quad \boldsymbol{a}_{\lambda}^{T_{i}} \in \mathcal{F}_{T_{i}} .
$$

Models that satisfy this relation seem to provide a natural class that essentially spans the full class of HJM models. One can frame this assertion as a formal theorem, but, since our goal here is to understand the nature of the LIBOR convexity bias, it seems sufficient just to restrict attention to models that have the LIBOR-rate representation (15).

\section{Estimation and the Inner Product Criterion}

Now we consider the publicly available data from the British Bankers' Association (www.bba.org.uk) on twelve spot LIBOR rates with maturities that vary from one month to twelve months. These data and the representations (13) and (14), allow one to construct a direct check of the inner product condition (11).

Since we are concerned here with the Eurodollar futures which are written on 3-month LIBOR, we only need to verify the inner product condition when the maturity difference $\lambda_{2}-\lambda_{1}$ is equal to 90 days, or $1 / 4$ on the 360 -day financial year scale. In Table 1 we report the estimated signs of $\left|\boldsymbol{a}\left(\lambda_{1}\right)\right|^{2}-\boldsymbol{a}\left(\lambda_{2}\right)^{\top} \boldsymbol{a}\left(\lambda_{1}\right)$ for the period January 2000 to December 2004. These estimates are based on the formulas (13) and (14) with $K=20$. Estimates using values of $K$ equal to 10, 15, 25, 30, and 40 were also computed and they did not differ meaningfully from the estimates 
with $K=20$. The bottom line is that there is a strong empirical evidence that the inner product condition (11) holds for the time period under consideration.

It is therefore ironic that one finds that during the same time period the Futures Rate-Forward Rate inequality (9) is often violated. Specifically, from Table 2 and the column labeled $\% V$, one finds that for a substantial fraction of the time the futures rate is below the forward rate. Moreover, Table 2 also shows that the observed difference $\Delta$ between the rates is often large enough to be economically significant.

More specifically, for each contract for December, March, June and September for the years 2000 through 2004, we considered the futures 3-month LIBOR rates that are implied by Eurodollar contracts. For each contract the futures rate process was observed for every business day for last nine months of the contract, and for each business day an associated forward LIBOR rate was computed from the BBA LIBOR data. These rate computations were done using the cubic spine interpolation method that has become traditional since Muelbroek (1992) and Grinblatt and Jegadeesh (1996). Here, to guarantee that our rate estimates are conservative, we used the highest intra-day futures rate instead of closing futures rate for each day.

What one finds from these computations is that even when the highest futures rate is compared to the corresponding forward rate, the futures rate is often below the forward rate. This observation squarely contradicts the conventional presumption that in theory and in practice one should have a positive value for the forward rate futures rate bias, $F_{\lambda}(t, T)-L_{\lambda}(t, T)$.

From Table 2, one also sees that the Eurodollar futures contract that expired in September 2003 was particularly extreme. For this contract, both the mean and median of the difference are negative. Moreover, the size of the negative bias seems to be quite substantial, although at present there is not any reliable method for assigning standard errors to these estimates. Still, for one measure of scale, one should note that Eurodollar futures prices are quoted with precision of 0.0025 , and, for another sense of scale, one can consider the oscillations of Figure 1 which gives the time series for $\Delta$ for the September 2003 contract. 
Taken together, Tables 1 and 2 present something of a paradox. In Table 2 we have 2783 observations of futures/forward differences and for $49 \%$ of these the difference is negative. Since the inner product condition (11) is a sufficient condition for a positive bias, one might reasonably expect to see a similar frequency of violations of the inner product condition (11). To explore this possibility, we computed 563 realized values of the difference $\left|\boldsymbol{a}\left(\lambda_{1}\right)\right|^{2}-\boldsymbol{a}\left(\lambda_{2}\right)^{\top} \boldsymbol{a}\left(\lambda_{1}\right)$. Here one should note that these differences that can be computed without any interpolation; that is, only observed LIBOR futures prices are needed for the computation. Surprisingly, in only $2 \%$ of the cases was there a positive difference.

\section{Concluding Remarks}

The inner product condition (11) has been found to hold up to empirical scrutiny for a rich (essentially complete) class of HJM models, and the inner product condition is sufficient for the positivity of the Futures Rate Forward Rate bias (8). For modelers and practitioners who rely upon the intuitive positivity of the bias this offers some theoretical assurance. Still, this reassurance is wrapped in a paradox, since it remains an empirical fact that the conventional bias inequality (8) often violated.

One possible resolution of the paradox is that the HJM term structure model is inadequate when applied to the Eurodollar futures market. A second, perhaps less likely resolution is that LIBOR futures offer as yet unexplained arbitrage possibilities. To choose between these two possibilities, or to yet other alternatives, would take us much further than we can go here.

\section{REFERENCES}

[1] G. Burghardt and B. Hoskins, "A question of bias," Risk 8, 63-70, 1995.

[2] M. Grinblatt and N. Jegadeesh, "Relative Pricing of Eurodollar Futures and Forward Contracts," Journal of Finance, 51, 1499-1522, 1996.

[3] A. Gupta and M. Subrahmanyam, "An empirical examination of the convexity bias in the pricing of interest rate swaps," Journal of Financial Economics 55, 239-279, 2000.

[4] D. Heath, R. Jarrow, and A. Morton, "Bond pricing and the term structure of interest rates. A new methodology for contingent claim valuation," Econometrica 60, 77-105, 1992.

[5] M. Henrard, "Eurodollar futures and options: convexity adjustment in HJM one-factor model," Working paper, 2005. 


\begin{tabular}{|c|c|c|}
\hline \multirow{2}{*}{$\begin{array}{c}\text { Maturities } \\
\left(\lambda_{1}, \lambda_{2}\right)\end{array}$} & \multicolumn{2}{|c|}{ Estimated difference $\left|\boldsymbol{a}\left(\lambda_{1}\right)\right|^{2}-\boldsymbol{a}\left(\lambda_{2}\right)^{\top} \boldsymbol{a}\left(\lambda_{1}\right)$} \\
\hline & Non-positive & Positive \\
\hline$(1 / 12,4 / 12)$ & 59 & 4 \\
\hline$(2 / 12,5 / 12)$ & 61 & 2 \\
\hline$(3 / 12,6 / 12)$ & 62 & 1 \\
\hline$(4 / 12,7 / 12)$ & 61 & 2 \\
\hline$(5 / 12,8 / 12)$ & 62 & 1 \\
\hline$(6 / 12,9 / 12)$ & 63 & 0 \\
\hline$(7 / 12,10 / 12)$ & 62 & 1 \\
\hline$(8 / 12,11 / 12)$ & 63 & 0 \\
\hline$(9 / 12,12 / 12)$ & 63 & 0 \\
\hline
\end{tabular}

Table 1. Inner Product Condition Checks for 63 Time Periods from Jan 2000 to Dec 2004 (Each period is 20 business days.)

Futures Forward Difference for EDU03

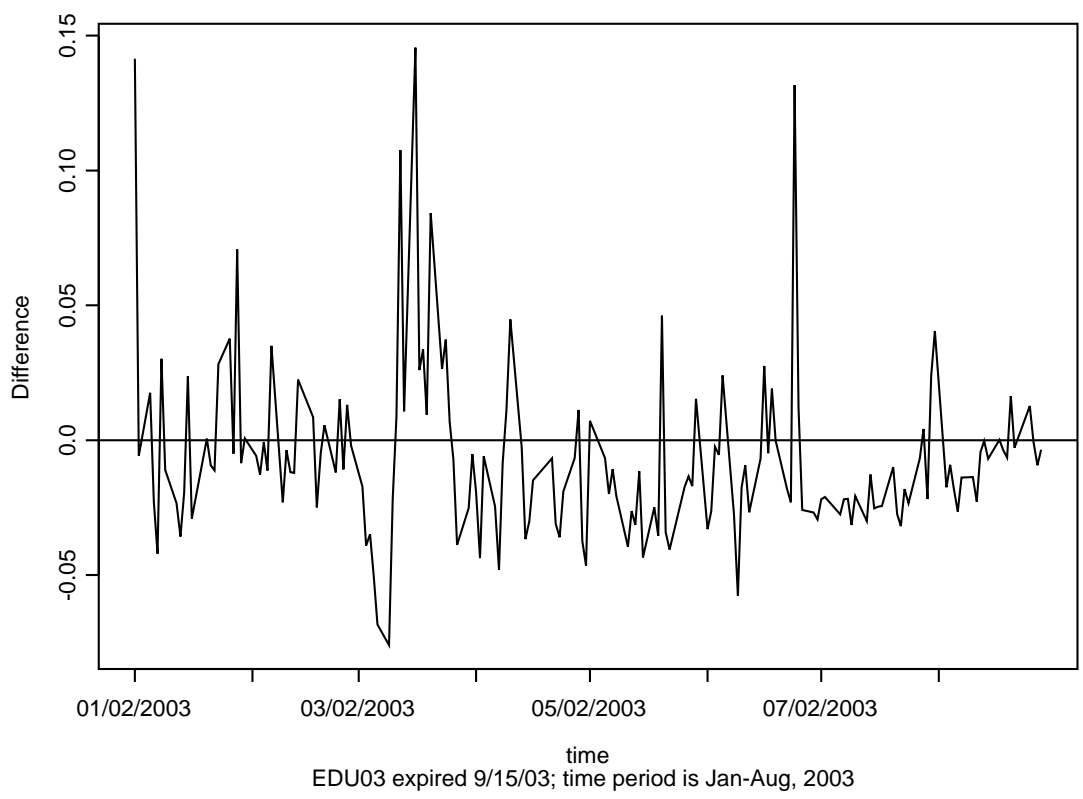

Figure 1. The Futures Rate/Forward Rate Difference $\Delta$ for EDU03 (EDU03 expired 9/15/03; time period is Jan-Aug, 2003) 
[6] I. Karatzas and S.E. Shreve, Brownian Motion and Stochastic Calculus, 2nd Edition, Springer, New York, 1991.

[7] I. Karatzas and S.E. Shreve, Methods of Mathematical Finance, Springer, New York, 1998.

[8] Meulbroek, L., "A comparison of forward and futures prices of an interest-sensitive financial asset," Journal of Finance, 47, 381-396, 1992.

[9] M. Musiela and M. Rutkowski, Martingale Methods in Financial Modelling, Springer, New York, 1997.

[10] V. Piterbarg and M. Renedo, "Eurodollar futures convexity adjustments in stochastic volatility models," Journal of Computational Finance 9, 71-94, 2006.

[11] Pozdnyakov, V. and Steele, J.M., "Convexity Bias in the Pricing of Eurodollars Swaps,", Methodology and Computing in Applied Probability, 4, 181-193, 2002.

[12] V. Pozdnyakov and J.M. Steele, "On the Martingale Framework for Futures Prices," Stochastic Processes and Their Applications, 109, 69-77, 2004.

[13] J.M. Steele, Stochastic Calculus and Financial Applications, New York, Springer, 2001. 


\begin{tabular}{l|c|c|cc|cc}
\hline Contract & Obs & $\% \mathrm{~V}$ & Mean $\Delta$ & $\operatorname{Std} \Delta$ & Median $\Delta$ & MAD $\Delta$ \\
\hline \hline EDZ00 & 166 & 18.07 & .0422 & .0667 & .0304 & .0418 \\
EDH01 & 164 & 42.07 & .0084 & .0419 & .0045 & .0350 \\
EDM01 & 164 & 42.07 & .0086 & .0418 & .0054 & .0357 \\
EDU01 & 166 & 63.86 & -.0071 & .0403 & -.0091 & .0349 \\
EDZ01 & 165 & 34.55 & .0255 & .0615 & .0180 & .0514 \\
EDH02 & 163 & 37.42 & .0175 & .0465 & .0095 & .0282 \\
EDM02 & 163 & 34.97 & .0257 & .0501 & .0118 & .0378 \\
EDU02 & 162 & 47.53 & .0083 & .0410 & .0029 & .0338 \\
EDZ02 & 163 & 46.63 & .0056 & .0483 & .0041 & .0383 \\
EDH03 & 164 & 58.54 & .0066 & .0459 & -.0098 & .0264 \\
EDM03 & 162 & 65.43 & -.0048 & .0406 & -.0109 & .0299 \\
EDU03 & 164 & 73.78 & -.0066 & .0323 & -.0112 & .0201 \\
EDZ03 & 164 & 69.51 & -.0050 & .0284 & -.0117 & .0198 \\
EDH04 & 163 & 61.34 & .0009 & .0298 & -.0032 & .0150 \\
EDM04 & 163 & 52.76 & .0090 & .0352 & -.0030 & .0223 \\
EDU04 & 164 & 54.27 & .0038 & .0403 & -.0058 & .0334 \\
EDZ04 & 163 & 33.13 & .0202 & .0501 & .0126 & .0297 \\
\hline
\end{tabular}

Table 2. The Percentage $\% V$ of Observations that Violate the Futures Rate-Forward Rate Inequality and Features of the Difference $\Delta$ Between the Rates 